\title{
Gewagt, geeint, gemeistert
}

\section{Christoph Bosshard}

Dr. med., Vizepräsident der FMH, Departementsverantwortlicher Daten, Demographie und Qualität DDQ

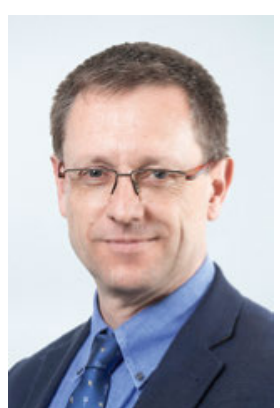

Alle sprechen von Leitlinien, koordinierter Versorgung und Interprofessionalität. Die FMH spricht nicht nur davon, sondern lebt sie vor mit dem SAQM-Projekt des interprofessionellen sektorenübergreifenden Behandlungspfads Kolorektalkarzinom. Es ist der SAQM gelungen, 20 Fachorganisationen, wovon die Hälfte nichtärztliche Verbände, für dieses herausfordernde Projekt zu gewinnen und einen Konsens zu finden. Es folgt ein kurzer Rückblick auf den begangenen Weg. Sind wir denn des Wahnsinns? - fragten wir uns, nachdem wir zur Vorbereitung dieses Projekts die internationale Literatur studiert und darauf aufbauend Expertengespräche geführt hatten. Ein solcher interprofessioneller und gleichzeitig sektorenübergreifender Behandlungspfad wurde nicht nur als internationale Pioniertat gesehen, sondern auch als zu anspruchsvolles Vorhaben. Dennoch haben wir es gewagt. Denn: «Wenn du schnell gehen willst, dann gehe alleine. Wenn du weit gehen willst, geh mit anderen" (afrikanisches Sprichwort). Und es hat sich gelohnt: gewonnen haben wir eine internationale Auszeichnung im Rahmen des European Clinical Pathway Congress und eine wertvolle Gemeinschaft mit den beteiligten Organisationen.

Wenn du schnell gehen willst, dann gehe alleine. Wenn du weit gehen willst, geh mit anderen.

Die initialen Umfragen haben uns einen Überblick der bereits existierenden Behandlungs- und Abklärungsschemata in diversen Regionen oder Institutionen ermöglicht in Richtung unseres Projekts. Nach intensiver Koordinationsarbeit und Konsensfindung liegt er nun vor: der interprofessionelle Behandlungspfad Kolorektalkarzinom. In der aktuellen Ausgabe finden Sie einen ausführlichen Bericht dazu.

Diesen Meilenstein gilt es nicht nur zu würdigen, sondern den beteiligten Personen und Organisationen herzlich zu danken. Für ihren Mut und Durchhaltewillen, mit uns diesen Weg zu beschreiten. Für ihre wertvolle Arbeit und ihr Wissen, das sie eingebracht haben, und für die nach wie vor beeindruckende Motivation und Initiative, den Weg weiterzugehen. Das ist nicht selbstverständlich, wenn man noch den bisher getätigten Aufwand und den Einsatz bedenkt: alle nicht vergüteten Stunden der Projektteammitglieder sowohl der Leistungserbringerorganisationen als auch der FMH. Dabei handelt es sich um einen Betrag von zirka CHF 700 000. Eine grosse Anerkennung auch dafür. Als Nächstes gilt es herauszufinden, was mit der Einführung eines solchen Behandlungspfads bewirkt wird. Verbessert sich die Früherfassung, Abklärung und Therapie? Führt dies zu einer Verteuerung, gleich-

\section{Die im Behandlungspfad definierten Key- Interventionen müssen in die Ausgestaltung der kantonalen Datensätze einfliessen.}

bleibenden Kosten oder sparen wir Ressourcen aufgrund eines koordinierteren und effizienteren Mitteleinsatzes? Bisher verfolgen wir die Hypothese, dass eine bessere Guideline-Adhärenz und Befolgung von Behandlungspfaden die Effizienz im Gesundheitswesen steigern. Nun wollen wir dies überprüfen. Dieses Vorhaben wird uns fordern, nicht nur ressourcenmässig. Auch hier braucht es auf allen Ebenen den Mut, Veränderungen anzupacken und zuzulassen. Die Projektleitung wird nun die nächsten Schritte konkretisieren. Ich zähle weiter auf unsere bewährte interprofessionelle Gemeinschaft, aber erwarte nun auch die Unterstützung seitens der Kostenträger, der Verwaltung und der Politik. Die zu schaffenden Rahmenbedingungen umfassen eine gesetzeskonforme Tarifierung der im Behandlungspfad definierten Leistungen und die Berücksichtigung der im Behandlungspfad definierten Key-Interventionen in der Ausgestaltung der kantonalen Datensätze der Krebsregister.

Wir haben heute Grund zum Feiern, aber morgen liegen noch viele Herausforderungen vor uns. Gemeinsam werden wir es schaffen! Ich danke allen - insbesondere den 20 beteiligten Organisationen und Projektteammitgliedern -, die uns unterstützt haben und weiterhin unterstützen werden. 